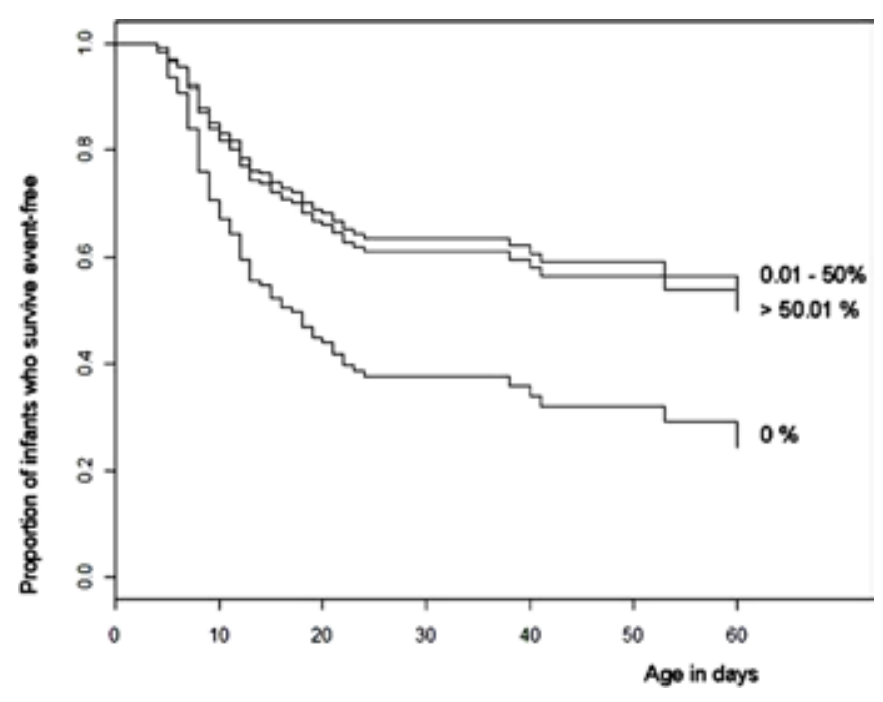

Abstract 227 Figure 1

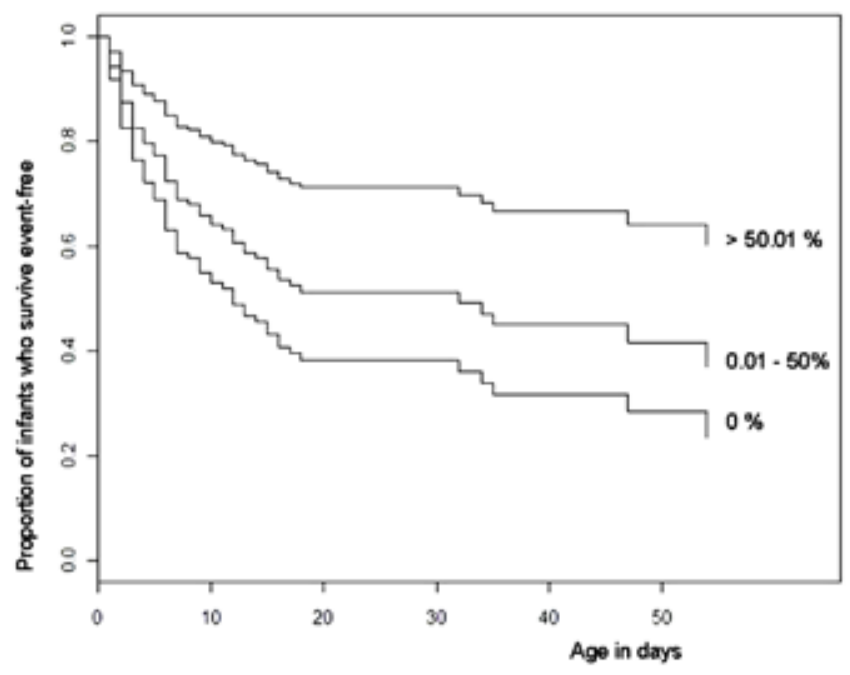

Abstract 227 Figure 2

\section{FACTORS ASSOCIATED WITH CONSTIPATION IN A PEDIATRIC INTENSIVE CARE UNIT}

doi:10.1136/archdischild-2012-302724.0228

1J Lopez, 'R Gonzalez, ${ }^{2} \mathrm{~J}$ Urbano, 'MJ Solana, ${ }^{2 M}$ Botran, ${ }^{2} \mathrm{~A}$ Garcia, ${ }^{1} \mathrm{~J}$ Lopez-Herce. ${ }^{1}$ Pediatric Intensive Care, Hospital General Universitario Gregorio Marañon. Instituto de Investigación Sanitaria Gregorio Marañon; 2 Pediatric Intensive Care, Hospital General Universitario Gregorio Marañon, Madrid, Spain

Backgrounds and aims Constipation is a frequent complication in critically ill patients although there are few studies in children.

Methods Prospective observational study with children admitted in the PICU for more than 3 days. PRISM, PELOD and PIM2 scores, total number of days with mechanical ventilation, duration and doses of sedatives and inotropes, and nutritional aims were recorded. Correlations between these parameters and time to first defecation were analyzed.

Results 84 children (median 13 months-old) were included. Mean time to first defecation was 4 days (range 0-19). A positive correlation with PRIMS (r:0.39), PELOD (r:0.33) and PIM2 (r:0.48) scores $(p<0.01)$ was observed. Moderate correlation existed between the time to first defecation with the administration of vecuronium $(\mathrm{r}: 0.40 \mathrm{p}<0.01)$ and the days without enteral feeding ( $r: 0.46$ $\mathrm{p}<0.01)$. There was a low correlation with days of mechanical ventilation $(r: 0.3 p<0.01)$, treatment with fentanyl $(r: 0.23 p<0.05)$, remifentanyl $(\mathrm{r}: 0.25 \mathrm{p}<0.05)$, and absolute and proportional gastric residual volume (r:0.24 $\mathrm{p}<0.05$ and $\mathrm{r}: 0.3 \mathrm{p}<0.01$, respectively). A negative association with daily enteral feeding volume (r:-0.37 $\mathrm{p}<0.01)$ was observed. Treatment with epinephrine or norepinephrine was also associated with a delay in the first defecation (5.6 vs 2.8 days $\mathrm{p}<0.01)$

Conclusions Mean time to first defecation in critically ill children is 4 days. Constipation in critically ill children seems to be associated with severity of illness. Enteral nutrition could help bowel motility.

\section{A RANDOMIZED PLACEBO CONTROLLED TRIAL OF BOVINE COLOSTRUM FED TO CHILDREN WITH SHORT BOWEL SYNDROME EVALUATED BY METABOLIC BALANCE STUDIES}

doi:10.1136/archdischild-2012-302724.0229

'L Aunsholt, ${ }^{2} \mathrm{~PB}$ Jeppesen, ${ }^{3} \mathrm{~N}$ Qvist, ${ }^{2 \mathrm{P}}$ Lund, ${ }^{4} \mathrm{PT}$ Sangild, ${ }^{4} \mathrm{~T}$ Thymann, 'S Husby. ${ }^{1}$ Department of Pediatrics, Hans Christian Andersen Childrens Hospital, Odense; ${ }^{2}$ Department of Medical Gastroenterology, Rigshospitalet, University Hospital of Copenhagen, Copenhagen; ${ }^{3}$ Department of Surgery, Odense University Hospital, Odense; ${ }^{4}$ Department of Human Nutrition, University of Copenhagen, Copenhagen, Denmark

Background and aims Parenteral support is indicated in short bowl syndrome (SBS) patients with intestinal failure to avoid metabolic imbalance, electrolyte and nutrient deficiencies, and to maintain adequate growth and function. Length, function and adaptation of residual bowel, promoted by e.g. luminal stimulation by nutrients determine the subsequent form of therapy. Colostrum contains putative stimulatory factors why, we hypothesise that supplementation could promote adaptation in children with SBS. Intestinal absorption of energy and wet weight was used to assess efficacy of colostrum and to define intestinal failure.

Methods and materials Nine children with SBS were included in a double-blinded randomised cross-over trial. Twenty percent of their enteral nutrition was replaced with bovine colostrum and a milk-mixture for 4 weeks, separated by a 4 week wash-out period. Between baseline and end of study, children were clinical and biological assessed 4 times.

Results Four HPN-patients had mean energy absorption of basal metabolic rate (BMR) of $81 \%$ and wet weight absorption of basal fluid need (BFN) of $6 \%$ at baseline compared to 5 non-HPN-patients with mean energy absorption of BMR of $196 \%$ and wet weight absorption of BFN at $76 \%, p=0.02, p=0.05$. Colostrum did not improved energy or wet weight absorption compared to milk-mix, $\mathrm{p}=0.85, \mathrm{p}=0.59$. Urea increased during colostrum supplementation, $\mathrm{p}=0.04$.

Conclusion The degree of intestinal function and a distinction between intestinal insufficiency and failure can successfully be assessed by energy and wet weight balance studies. Bovine colostrum did not promote intestinal adaptation.

\section{HYPOPHOSPHATEMIA: A RISK FACTOR FOR INSULIN REQUIREMENT IN ELBW INFANTS?}

doi:10.1136/archdischild-2012-302724.0230

'L Dreyfus, 1,2CJ Fischer, 3,4,5 D Maucort-Boulch, 1,6M Essomo Megnier Mbo Owono, 'S Laborie, 1,70 Claris. 'Department of Neonatology, Hôpital Femme Mère Enfant Hospices Civils de Lyon, Bron, France; ${ }^{2}$ Department of Neonatology, Centre Hospitalier Universitaire Vaudois et Université de Lausanne, Lausanne, Switzerland; ${ }^{3}$ Department of Biostatistics, Hospices Civils de Lyon, Lyon; ${ }^{4}$ CNRS UMR 5558. Equipe BiostatistiqueSanté, Laboratoire de Biométrie et Biologie Evolutive; ${ }^{5}$ Université Claude Bernard Lyon 1. Villeurbanne, France; ${ }^{6}$ Department of Neonatology, Centre Hospitalier de Libreville, Libreville, Gabon; 'EAM 4128, Université Claude Bernard Lyon 1, Villeurbanne, France 
Background and aims Insulin is frequently required to treat hyperglycemia that increases both mortality and morbidity in ELBW infants. Adult and animal studies suggest a link between hypophosphatemia and insulin resistance. Our objective was to define whether hypophosphatemia increases the risk of insulin requirement in ELBW infants.

Methods This observational study included ELBW infants admitted in our NICU between 01.01.2010 and 31.12.2011 who survived until DOL14. Laboratory and clinical data were retrospectively collected. According to the NICU policy, phosphatemia was measured before DOL3 and glycemia was checked daily during parenteral nutrition. Insulin was introduced in case of refractory hyperglycemia $>11 \mathrm{mmol} / 1$. Depending on the lowest phosphatemia before DOL3, patients were divided into hypophosphatemic (HP, $<1.2$ $\mathrm{mmol} / \mathrm{l})$ and controls $(\geq 1.2 \mathrm{mmol} / \mathrm{l})$. Uni- and multivariable analysis compared the time to insulin requirement using survival models.

Results In all, 126 patients were included: $39 \mathrm{HP}, 87$ controls. Mean(SD) gestational age was 27.8 (1.5) in HP and 27.4 (1.5) weeks in controls, birthweight was $770(140)$ and $837(109)$ grams. Insulin was required in 19/39 (49\%) HP and 26/87 (30\%) controls with a delay of 17 (10) and 22 (9) days respectively. The unadjusted hazard ratio of insulin requirement in HP was 1.93 (95\%CI: 1.07-3.49, $\mathrm{p}=0.03$ ). After adjustment for gestational age, birthweight, sex, IUGR and sepsis, the hazard ratio was still 1.6 (95\%CI: 0.86-3.17) but not significant $(\mathrm{p}=0.13)$.

Conclusion Hypophosphatemia may be a risk factor for insulin requirement in ELBW. Multivariable analysis shows that age and birthweight could also influence this outcome. Whether aggressive management of hypophosphatemia can improve glycemia control deserves to be studied.

\section{A RANDOMISED TRIAL OF VOLUME-TARGETED VERSUS PRESSURE-LIMITED VENTILATION IN PREMATURELY BORN INFANTS}

doi:10.1136/archdischild-2012-302724.0231

10 Chowdhury, '2 Peacock, 'GF Rafferty, 'S Lee, 'S Hannam, 'A Greenough. 'Division of Asthma, Allergy \& Lung Biology, MRC-Asthma UK Centre in Allergic Mechanisms of Asthma; 'Division of Health and Social Care Research, King's College London, London, UK

Background and aims Meta-analysis of randomised trials (RCTs) demonstrated that volume-targeted ventilation (VTV) in comparison to pressure-limited ventilation (IPPV) reduces BPD/death, pneumothorax, hypocarbia and PVL/grade 3-4 IVH in prematurely born infants. Certain RCTs, however, employed different ventilators in the two arms and, overall, a range of VT levels were used. Our aim was to undertake an RCT in prematurely born infants with acute respiratory distress comparing IPPV with VTV, using a VT level of $5 \mathrm{ml} / \mathrm{kg}$, which has been shown to reduce the work of breathing.

Methods Infants $<34$ weeks of gestational age and $<24$ hours of age were recruited. The primary outcome was the time taken to achieve pre-specified weaning criteria. Secondary outcomes included the occurrence of PDA, pneumothorax, IVH, PVL and hypocarbia; hypocarbia was defined as a $\mathrm{PaCO}_{2}$ of $<4.5 \mathrm{kPa}$ on any blood gas in the first 72 hours after birth. Infants met failure criteria if they required $\mathrm{HFO}$, peak pressures $>26 \mathrm{~cm} \mathrm{H}_{2} \mathrm{O}$ or had a pulmonary haemorrhage. Analysis was by intention-to-treat.

Results The planned sample size of 40 infants was achieved, with no significant differences in the two groups' demographics. The time taken to achieve weaning criteria was similar in the two groups [14 hours (VTV) versus 23 hours (IPPV); hazard ratio=0.82 (95\% CI $0.42,1.58)], p=0.55$. Five "VTV" and three "IPPV" infants met failure criteria, $p=0.69$. Fewer "VTV" than "IPPV" infants had hypocarbia (8 versus 19), $\mathrm{p}<0.001$.
Conclusion VTV was associated with a significantly lower incidence of hypocarbia.

\section{THE TIMING OF SURFACTANT PROPHYLAXIS IN VERY-LOW- BIRTH-WEIGHT PRETERMS: IS EARLIER BETTER?}

doi:10.1136/archdischild-2012-302724.0232

E Okulu, S Arsan, IM Akin, A Kilı̧, S Alan, B Atasay. Department of Pediatrics, Division of Neonatology, Ankara University, Ankara, Turkey

Aim To determine whether the immediate bolus strategy treatment could decrease the subsequent need for ventilation compared to the administration of surfactant prophylaxis at 15-minutes.

Methods All infants born before 29 weeks', and infants born at 29 to 30 weeks' without antenatal steroid(ANS) were randomized. Infants of group-1 were intubated immediately after birth, of group- 2 received standard resuscitation measures, than were intubated at 15 -minutes. All received $100 \mathrm{mg} / \mathrm{kg}$ surfactant. During these managements infants were ventilated with T-piece(NeoPuff). Then infants were extubated to NCPAP(Infant Flow ${ }^{\circledR}$ ) if respiratory drive was present. The primary outcome was the need for MV within the first 3-days of life. The secondary outcomes were neonatal morbidities, mortality and duration of hospitalization.

Results Total of 80 newborns were enrolled (fourty infants in each group). Prenatal and natal features were similar in groups. Ten infants in group-1, 13 infants in group-2 couldn't be extubated after surfactant. GA and BW of them were lower than the extubated infants. Six infants in group-1, four infants in group-2 needed MV during the first 3-days. Total respiratory support duration was lower in group-1. There were no significant differences between the groups with a respect to PDA, NEC, IVH, sepsis, ROP, BPD, mortality and duration of hospitalization.

Conclusion Our study didn't demonstrate a superiority of the immediate bolus strategy of surfactant prophylaxis combined with early-NCPAP to the administration of surfactant at 15-minutes after birth with early-NCPAP. Surfactant prophylaxis at-15 minutes with early-NCPAP seems to be sufficiently effective to yield favorable outcomes in small preterm infants.

\section{MECHANICAL VENTILATION-INDUCED APOPTOSIS IN NEWBORN RAT LUNG IS MEDIATED VIA FASL/FAS PATHWAY}

doi:10.1136/archdischild-2012-302724.0233

1,2A Kroon, ${ }^{2}$ V Del Riccio, ${ }^{2} \mathrm{~T}$ Tseu, ${ }^{2} \mathrm{Z}$ Huang, ${ }^{2} \mathrm{~J}$ Wang, ${ }^{2} \mathrm{M}$ Post. ${ }^{2}$ Erasmus MC - Sophia, Rotterdam, The Netherlands; 'Lung Biology Research, Physiology and Experimental Medicine Program, Hospital for Sick Children Research Institute, Toronto, ON, Canada

Rationale Mechanical ventilation induces pulmonary apoptosis and inhibits alveolar development in preterm infants, but the molecular basis for this apoptotic injury is unknown.

Objective To determine the signaling mechanism(s) of ventilation(stretch)-induced apoptosis in newborn rat lung.

Methods Seven-day old rats were ventilated with room air for 24 $\mathrm{h}$ using moderate tidal volumes $\left(8.5 \mathrm{~mL} . \mathrm{kg}^{-1}\right)$. Isolated fetal rat lung epithelial and fibroblast cells were subjected to continuous cyclic stretch (5, 10 or $17 \%$ elongation) for up to $12 \mathrm{~h}$.

Measurements and main results Prolonged ventilation increased significantly the number of apoptotic alveolar type II cells (i.e. TUNEL-labelling, anti-cleaved caspase- 3 immunochemistry) and was associated with increased expression of the apoptotic mediator Fas Ligand (FasL). Fetal lung epithelial cells, but not fibroblasts, subjected to maximal (i.e. $17 \%$, but not lesser elongation) cyclic stretch exhibited increased apoptosis (i.e. nuclear fragmentation; DNA laddering) which appeared to be mediated via the extrinsic pathway (increased expression of FasL and cleaved caspase-3, -7 and -8). The intrinsic pathway appeared not to be involved (minimal mitochondrial 